\title{
PREPARACIÓN DE ADSORBENTES TERMOÁCIDOS A PARTIR DE BENTONITAS DE LA REGIÓN CENTRAL PARA SU EMPLEO EN LA RETENCIÓN DE METALES PESADOS
}

\author{
PREPARATION OF TERMOACID ADSORBENT OF THE CENTRAL REGION BENTONITAS \\ FOR THEIR EMPLOYMENT IN THE RETENTION OF HEAVY METALS
}

\author{
Pascual Guevara Yanqui ' Jesús Montoya Torres'
}

\section{RESUMEN}

El trabajo se desarrolló teniendo en cuenta la gran importancia que implica la protección del medio ambiente $\mathrm{y}$ en particular el tratamiento de las aguas industriales contaminadas con metales pesados, los cuales representan un gran peligro para la salud. El empleo de la arcilla modificada como adsorbente efectivo para la remoción de metales pesados, plomo, zinc, cobre y hierro. El proceso de adsorción se realizó a escala batch tomando en consideración las variables como concentración de metal a adsorber, tiempo de adsorción, cantidad de adsorbente. Se encontró que las arcillas modificadas son efectivas removiendo entre el 82,00 98,98 \% de iones plomo de soluciones con concentraciones iniciales de 10 $\mathrm{mg} / \mathrm{L}$ de plomo, también se comprobó que hay una buena adsorción de hierro y en menor medida la adsorción de zinc, con concentraciones iniciales de $10 \mathrm{mg} / \mathrm{L}$ de cada metal. Con estas experiencias se comprobó que las arcillas sometidas a un tratamiento termoácido mejoran sus características estructurales y texturales que se hacen adecuadas para la adsorción de metales, en este caso plomo, zinc, cobre y fierro.

Palabras clave: Arcilla modificada, metales pesados, adsorción, tratamiento termoácido.

\begin{abstract}
This study was developed bearing in mind the big importance that implies the protection of the environment and in particular the treatment of the industrial waters contaminated with heavy metals, which represent a big danger for health. The employment of modified clay as cash adsorbent for the removal of heavy metals, lead, zinc, copper and iron. The process of adsorbing was realized to scale batch taking in consideration the variables as metal concentration to adsorb, time of adsorbing, adsorbent quantity. It wasfound that the modified clays are effective removing between $82,00 \quad 98,98 \%$ of ions lead of solutions with initial concentrations of $10 \mathrm{mg} / \mathrm{L}$ of lead, also it was proved that there is a good adsorbing of iron and in minor measurement the adsorbing of zinc, with initial concentrations of $10 \mathrm{mg} / \mathrm{L}$ of every metal. With these experiences it been proved that the clays submitted to a treatment term acid improve.
\end{abstract}

Key words: Modified clay, heavy metals, adsorbing, treatment term acid 


\section{INTRODUCCIÓN}

La investigación tuvo como objetivo desarrollar un proceso para obtener un adsorbente termoácido partiendo de una arcilla natural del tipo bentonita (montmorillonita) para utilizar en la retención de metales pesados presentes en aguas industriales contaminadas.

La Región Central del país, sobre todo el Valle del Mantaro, es una zona muy rica en recursos no metálicos, tenemos yacimientos de arcillas en San Juan de Jarpa, Mitopampa, Pucará, entre otros; destinados solamente para la obtención de cerámicos. Los objetivos fueron, desarrollar un proceso de retención de metales pesados presentes en aguas contaminadas; y determinar las mejores condiciones de operación para la obtención del adsorbente.

\section{MATERIAL Y MÉTODOS}

Se recolectaron dos muestras de arcillas de la región, una proveniente de Chupuro (muestra $A$ ) y otra de San Juan de Jarpa (muestra B). La primera arcilla presenta una coloración rojiza; la segunda presenta una coloración clara, estas muestras fueron secadas a $100^{\circ} \mathrm{C}$ por una hora para luego ser sometidas a un proceso de molienda y tamizado a malla Tyler $\mathrm{N}^{\circ} 60$.

Se hicieron las siguientes pruebas cualitativas; la primera fue la determinación de carbonatos, para esto se empleó ácido acético y ácido clorhídrico; se pesó 1 gramo de muestra y se le agregó $1 \mathrm{~mL}$ de cada uno de los ácidos. Para la presencia de materia orgánica, se tomó un gramo de muestra y se le agregó $5 \mathrm{~mL}$ de agua oxigenada, en la sección sedimentables; se pesó 10 gramos de muestra y se disolvió en un vaso de $250 \mathrm{~mL}$ con $100 \mathrm{~mL}$ de agua y se agitó por una hora, luego se trasvasó la solución a una probeta de $100 \mathrm{~mL}$ y se dejó sedimentar por dos horas. Para el $\mathrm{pH}$, se separó $2 \mathrm{~g}$ de arcilla; se le agregó en agitación constante $100 \mathrm{~mL}$ de agua destilada hasta formar una suspensión estable y se introdujo el peachímetro previamente calibrado.

\section{Activación}

En esta etapa la arcilla seleccionada (B) previamente secada y pulverizada fue sometida a la acción del ácido clorhídrico $1 \mathrm{~N}$ (relación arcilla/acido 1/10) en un reactor con reflujo mantenido en agitación y temperatura constante de $90^{\circ} \mathrm{C}$ a la presión atmosférica durante dos horas. El material obtenido se lavó con agua destilada hasta llevar el $\mathrm{pH}$ a un valor de 4, luego el material fue secado y calcinado a $200^{\circ} \mathrm{C}$ por dos horas.

\section{Proceso de adsorción}

Se prepararon soluciones estándares de $10 \mathrm{mg} / \mathrm{L}$ de plomo, zinc, cobre y fierro respectivamente, a partir de las soluciones patrón de $1000 \mathrm{mg} / \mathrm{L}$ de cada uno de los elementos mencionados; estas soluciones se usaron para las pruebas de adsorción; luego se preparó una solución combinada de los elementos mencionados con una concentración de $10 \mathrm{mg} / \mathrm{L}$ de cada elemento para su respectiva prueba adsorción; también se realizaron las diluciones para construir las curvas de calibración de cada elemento.

El proceso de adsorción se realizó en sistema batch, tanque de agitación, los parámetros de operación que se tuvieron en cuenta fueron: tamaño de partícula, concentración del adsorbente, revoluciones por minuto, concentración de metal presente, $\mathrm{pH}$ inicial de operación.

\section{RESULTADOS}

Después del análisis cualitativo (tabla 1), se puede ver que las arcilla $B$ presenta buenas características para ser activada como recomienda Fayad [1].

I ahla 1. I)etermınarıñn rje rarhnnatns.

\begin{tabular}{|c|c|c|}
\hline Muestras & $\mathbf{\Lambda}$ & $\bar{B}$ \\
\hline Acido asct ca & Prconcia de $\supset 02$ & No nay roas cićn \\
\hline A cido cloth dri: 0 & Presencia de 2.02 & No oay reac cićn \\
\hline A gua oxlcenada & INo ha' reac:iśn & No רay reacclín \\
\hline $\begin{array}{l}\text { Oedimentables } \\
\mathrm{pH}\end{array}$ & Muy poe 0 & $\begin{array}{l}\text { Major parte } \\
9.01\end{array}$ \\
\hline
\end{tabular}

El análisis estructural, se trabajó con la arcilla $\mathrm{B}$, la identificación de los grupos superficiales de la muestra B se realizó por espectroscopia Infrarroja

Figura 1: Espectrograma IR de la arcilla B

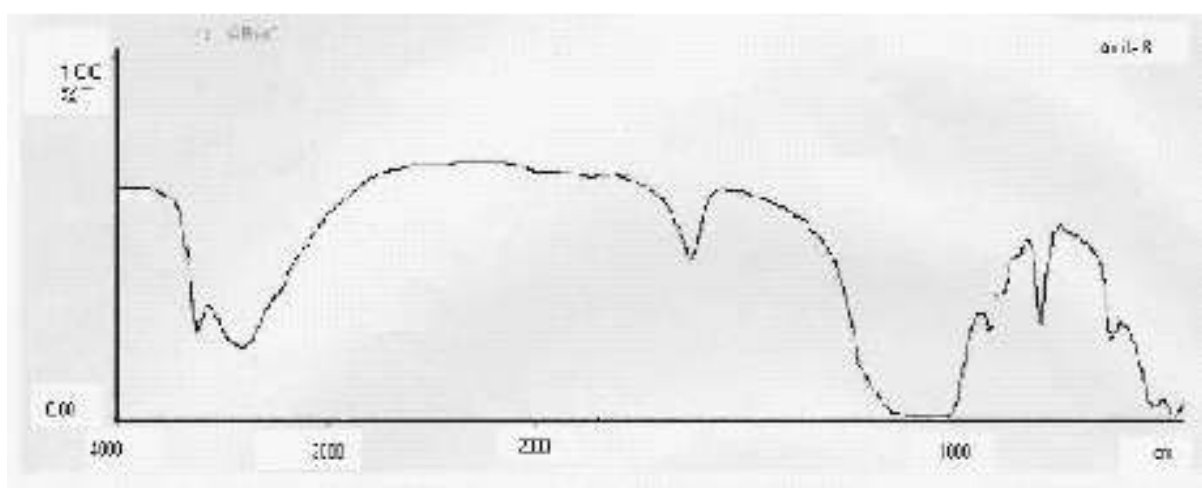


En la figura 1, se pueden observar vibraciones de tensión y de deformación a $3628 \mathrm{~cm}^{-1}$ y $1636 \mathrm{~cm}^{-1}$ correspondientes a los enlaces de hidrógeno entre Inhla th llatos para tiu el agua interlaminar y los oxígenos superficiales. A $1040 \mathrm{~cm}^{-1}$ y $521 \mathrm{~cm}^{-1}$ aparecen vibraciones de tensión de enlace Si-O-Si y de deformación del Si-O respectivamente. La señal correspondiente a la sílice libre se presenta a $795 \mathrm{~cm}^{-1} \mathrm{y}$ la de enlace Si-O-Mg a 468 $\mathrm{cm}^{-1}$.

\begin{tabular}{cc}
$\begin{array}{c}\text { Cone CII } \\
\text { (jpui) }\end{array}$ & (imin) \\
\hline 10 & 0 \\
6.3 & 1 \\
4.68 & 2 \\
3.92 & 3 \\
3.36 & 4 \\
3.15 & 5 \\
2.77 & 10 \\
2.7 & 15 \\
2.07 & 30 \\
379 & 60 \\
4.34 & 240 \\
4.34 & 2110 \\
\hline
\end{tabular}

\section{Curvas de calibración:}

A partir de soluciones estándar se construyeron las curvas de calibración, de los cuales se platean las ecuaciones lineales correspondientes:
Cobre: Concentración Inicial 10 ppm Cu, Agitación 450 rpm, malla ASTM 30-40.

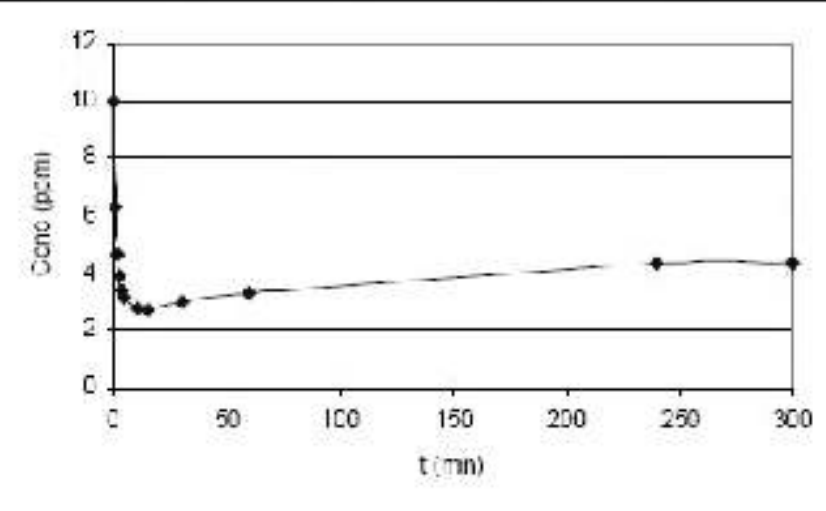

Fig. 3 Adsurciún de Cobre

Zinc: Concentración Inicial 10 ppm Zn, Agitación 450 rpm; malla ASTM 30-40.

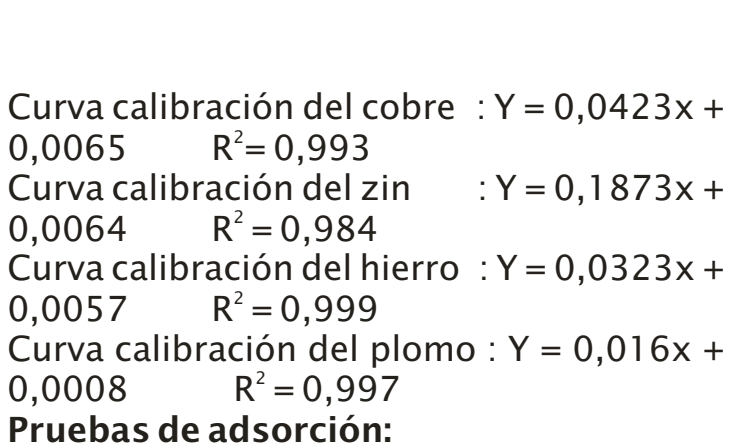

Se realizaron las siguientes pruebas de adsorción ( $3 \mathrm{~g}$ de adsorbente en $300 \mathrm{~mL}$ de solución):

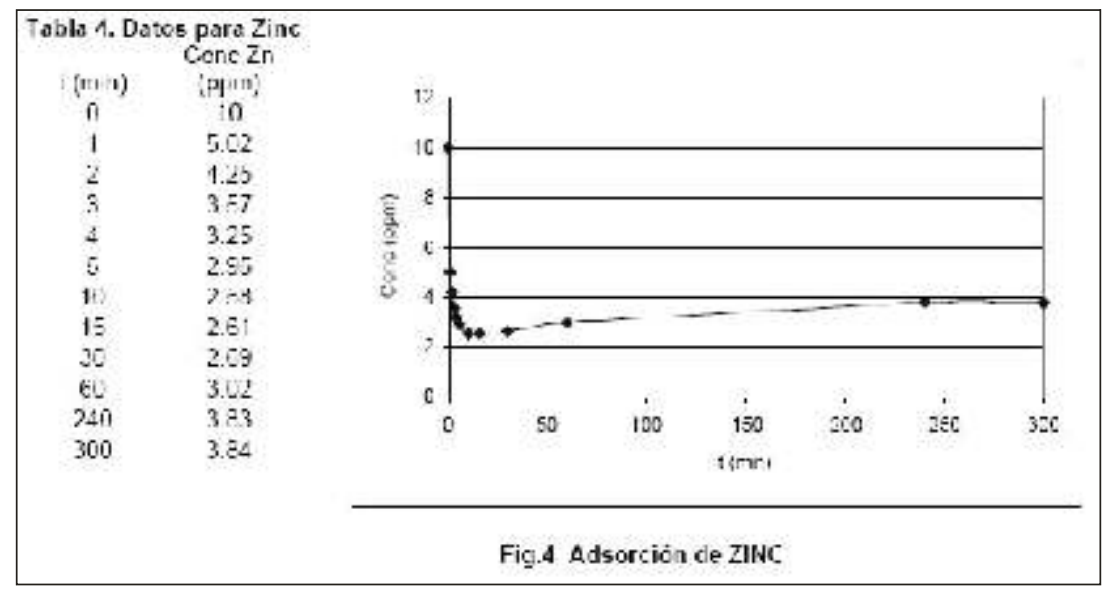

Plomo: Concentración Inicial 10 ppm Pb, Agitación 450 rpm, malla ASTM 30-40.

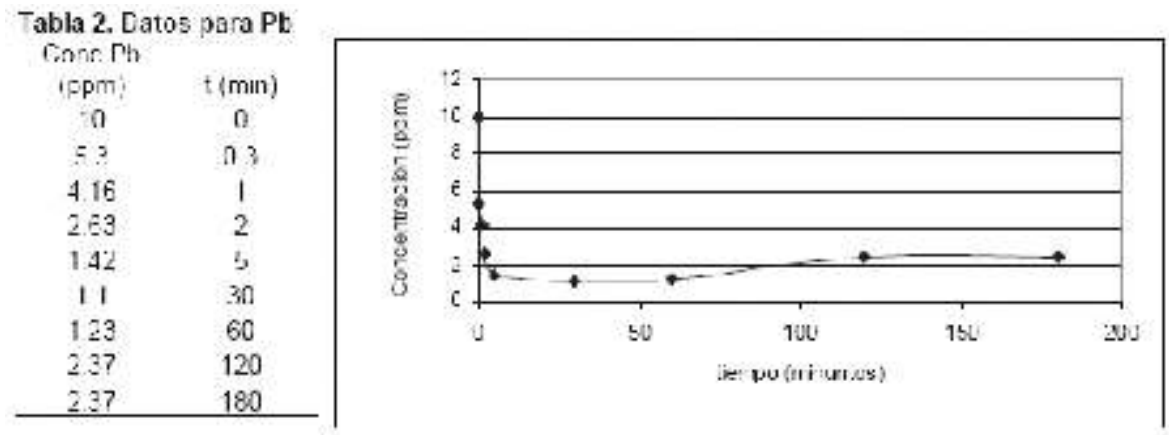

Fig̣ 2. Adsorciōn de Plomo 
Fierro: Concentración Inicial 10 ppm Fe, Agitación 450 rpm, malla ASTM 30-40. agitación y malla ASTM 30-40 para el tamaño del adsorbente.

\begin{tabular}{|c|c|}
\hline$t(\min )$ & $\begin{array}{c}\text { Conc Fe } \\
\text { (ppm) }\end{array}$ \\
\hline U & iv \\
\hline 1 & E.1 \\
\hline 2 & 284 \\
\hline 3 & 1.67 \\
\hline 4 & 1.34 \\
\hline 5 & 1.13 \\
\hline 10 & $0 \Omega 5$ \\
\hline 15 & 075 \\
\hline 30 & 073 \\
\hline fin & $0>n$ \\
\hline 240 & 0.94 \\
\hline 320 & 0.91 \\
\hline
\end{tabular}

Adsorción de los metales combinados en una solución: Concentración Inicial 10 ppm , Fe, 10 ppm Zn, 10 ppm Cu, 10 ppm Fe, Agitación 450 rpm, 1 \% de adsorbente malla ASTM 30-40.

\section{ABLA N"6 AUSUIRCION DE ME ALLS COMBINAUUS}

\begin{tabular}{|c|c|c|c|c|}
\hline$t$ (mir) & $\begin{array}{l}\text { Conr. } P h \\
\text { (ppn) }\end{array}$ & $\begin{array}{c}\text { Conr.7n } \\
\text { (pem) }\end{array}$ & $\begin{array}{l}\text { Cone CI } \\
\text { (opm) }\end{array}$ & $\begin{array}{l}\text { Sinnc } \Gamma_{e} \\
\text { (ppr) }\end{array}$ \\
\hline 0 & 10 & 10 & 10 & 10 \\
\hline 0.3 & 7.98 & 5.88 & 9.54 & 7.24 \\
\hline 1 & 1.34 & $5.6 \mathrm{~J}$ & Y.03 & 3.12 \\
\hline 2 & fin 89 & 5,5 & 766 & 471 \\
\hline 5 & 6.01 & 5.32 & $7 . C 5$ & 2.73 \\
\hline 0 & 5.94 & 5.25 & 6.81 & 2.63 \\
\hline is & b. 16 & b.2. & 6.12 & 2.03 \\
\hline 30 & G.07 & 5.22 & 0.02 & 2.02 \\
\hline 60 & 6.13 & 5.23 & 6.91 & 2.11 \\
\hline 120 & 6.13 & 5.29 & 7 & 2.11 \\
\hline 180 & 7.21 & 5.33 & 7.53 & 2.6 \\
\hline 300 & 7.21 & 5.85 & 7.65 & 2.6 \\
\hline
\end{tabular}

\section{DISCUSIÓN}

Cuando se realiza la adsorción de metales en forma individual se presenta mayor adsorción de plomo y fierro ( 89 y $92,7 \%$ respectivamente). Mientras que si se trabaja en una solución combinada con los cuatro metales se observó que el fierro y zinc son los metales que se retienen con mayor porcentaje ( 74 y $41,5 \%)$. Las condiciones de operación para las adsorciones serán de 1\% de adsorbente, 450 RPM de
Los resultados nos permiten hacer la interpretación siguiente: La activación ácida de la arcilla se debe realizar con ácido clorhídrico $1 \mathrm{~N}$ en un reactor de reflujo en relación de $1 / 10$ (arcilla/ácido) a $90^{\circ} \mathrm{C}$ por dos horas, la calcinación a $200^{\circ} \mathrm{C}$ por dos horas, se trabajó con la arcilla de San Juan de Jarpa por tener mejores características para procesarla como adsorbente, como lo indica el espectrograma de IR (fig. 1) y el DRX, se logró una retención del $89 \%$ de plomo en 30 minutos, una retención del $73 \%$ de Cobre en 15 minutos, se logro una retención de 73,9\% de zinc en 15 minutos, una retención de 92,7\% de fierro en 30 minutos, luego en una mezcla de $\mathrm{Pb}, \mathrm{Zn}, \mathrm{Cu}$ y Fe se logró en 300 minutos retener: $30 \%$ de $\mathrm{Pb}, 41,5 \%$ de Zn, 23,5 \%Cu y $74 \%$ de fierro.

\section{REFERENCIAS BIBLIOGRÁFICAS}

1. Fayad J. Evaluación de Arcillas Activadas como Adsorbentes de Efluentes industriales con Contenido de Cromo (Tesis de grado): Universidad Nacional de Ingeniería; 1998.

2. Hernández R, Rojas J. Empleo de Arcillas Modificadas en el Tratamiento Galvánico (Tesis de grado): Universidad Nacional de Ingeniería; 2001.

3. Sun Kou, M.R. .(Tesis Doctoral). Universidad de Complutense de Madrid; 1994.

4. Besoain N. Eduardo. Minerología de arcillas de suelo. México; 1978.

5. Zambrano D. Estudio de bentonitas y Principales Ocurrencias en el País. (Tesis de grado): Universidad Nacional Mayor de San Marcos; 1985.

6. Treybal R. Operaciones de Transferencia de Masa. México: Editorial Mc Graw Hill; 1980.

7. CONCYTEC. III Curso Taller Iberoamericano Sistemas Catalíticos para la Protección Ambiental. Lima; 2001.

E-mail: pascual_guevara@hotmail.com 